\title{
Analysis of a Computational Model of Dopamine Synthesis and Release through Perturbation
}

Cullen, M., \& Wong-Lin, K. (2014). Analysis of a Computational Model of Dopamine Synthesis and Release through Perturbation. In Unknown Host Publication IEEE. https://doi.org/10.1109/BIBM.2014.6999252

Link to publication record in Ulster University Research Portal

\section{Published in:}

Unknown Host Publication

Publication Status:

Published (in print/issue): 01/01/2014

DOI:

10.1109/BIBM.2014.6999252

\section{Document Version}

Author Accepted version

\section{General rights}

Copyright for the publications made accessible via Ulster University's Research Portal is retained by the author(s) and / or other copyright owners and it is a condition of accessing these publications that users recognise and abide by the legal requirements associated with these rights.

\section{Take down policy}

The Research Portal is Ulster University's institutional repository that provides access to Ulster's research outputs. Every effort has been made to ensure that content in the Research Portal does not infringe any person's rights, or applicable UK laws. If you discover content in the Research Portal that you believe breaches copyright or violates any law, please contact pure-support@ulster.ac.uk. 


\title{
Analysis of a Computational Model of Dopamine Synthesis and Release through Perturbation
}

\author{
Maell Cullen and KongFatt Wong-Lin \\ Intelligent Systems Research Centre, School of Computing and Intelligent Systems, \\ University of Ulster, Magee Campus, \\ Northland Road, Derry Londonderry, Northern Ireland, BT48 7JL, UK \\ k.wong-lin@ulster.ac.uk
}

\begin{abstract}
Dopamine is an important neurotransmitter responsible for regulating various brain functions such as learning and cognition. Dysfunctions within the dopaminergic system are implicated in many neurological and neuropsychiatric disorders. To understand such a complex system, biologically realistic multiscale computational models are necessary. Such models require the extraction of relevant and important factors or processes from one scale to bridge and interact with systems at other scales. In this paper, we analyze an influential computational model of dopamine synthesis and release within a pre-synaptic terminal by systematically perturbing its variables/substrates. Based on the relative changes in steady states and the time to reach the new perturbed steady states, we found that the substrates within the cascade of intracellular biochemical reactions can vary widely in terms of influence and timescale. We then categorize the substrates according to their relative timescales and changes in steady states. The perturbation results are then used to guide our selection for the most appropriate equations and functions to be approximated in developing reduced models of the original model. Our preliminary simulation results show that either a slow or fast version of the reduced model can be simulated significantly faster than the original model. Our work demonstrates, through perturbation analysis, the feasibility of reduced models of the dopaminergic presynaptic terminal to improve computational efficiency, implement in multiscale modelling, and in silico neuropharmacology.
\end{abstract}

Keywords-Mathematical model; dynamical systems; neuromodulator; neuropharmacology; multiple timescales

\section{INTRODUCTION}

Dopamine (DA) is a catecholamine neurotransmitter (neuromodulator) mediated by $\mathrm{G}$ protein-coupled dopamine receptors [1]. DA has been intensively studied due to its critical role in motor control, motivation, reward based behavior, hypertension and hormonal regulation [2]. Empirical studies have shown a correlation between the activity of phasic DA neuron activity and the temporaldifference (TD) reward prediction error of reinforcement learning, suggesting that DA neurons capable of encoding variance between anticipated and obtained awards through bursting and pausing patterns of firing [3]-[4].

Dysfunctions in the DA system are linked to a number of brain disorders, and a large number of pharmacologically active compounds that interact with dopamine receptor function have been developed and used clinically for the

K.F.W.-L. was partially supported by the Northern Ireland Functional Brain Mapping Project, funded by InvestNI and the University of Ulster. treatment of various disorders [5]-[9]. Moreover, drugs such as cocaine, amphetamines and L-DOPA (a DA precursor) can induce psychotic episodes by increasing dopamine levels [10]. Addictive drugs and natural rewards have a modulatory effect on DA signaling, producing changes in synaptic plasticity within the DA system and DA receptive neurons. Several features of addiction have been attributed to the DA system such as both short and long term changes in the firing of dopamine neurons in the ventral tegmental area [11] and a significant, long-term down regulation of DA D2 receptors in the striatum [12]. Hence, the DA system is a highly important target for neuropharmacology.

Indeed, the DA system has been the target of some effective pharmacological treatments for pathological conditions. Indirect DA receptor agonists, together with LDOPA, have been used to reduce symptom severity of Parkinson's disease patients with great efficacy [13]. Although DA replacement therapy serves only to alleviate symptoms and has not been known to treat Parkinson's disease, it has led to the discovery of more DA receptor agonists, a search that could be improved with the use of computational models. The DA system has also been the target of drugs prescribed for pituitary tumors [14], type 2 diabetes [15], depression and bipolar disorder [16].

Significant progress has been made in discovering the structural, genetic, physiology and pharmacological properties of dopamine neurons [17]. This has facilitated the development of sufficiently realistic computational models, for example, computational models of the DA neurons, DA synthesis, release and reuptake, and signal transduction [18] [20]. Biologically plausible computational models can assist in furthering our understanding of how neuromodulators contribute at the neuronal circuit and behavioral levels [21][24].

As neuromodulators act across multiple spatial and temporal domains, an important modelling approach is to develop and simulate multiscale models. However, multiscale models are currently still not as prevalent as approaches in genomics, molecular/cellular biology, pharmacokinetics and metabolism in the pharmaceutical industry [25]-[26]. An essential element of multiscale models is to extract relevant and important factors or processes at one scale (e.g. molecular) to bridge and interact with other scales (e.g. system) [27]. However, it is sometimes not immediately clear 
which factors or processes are relatively more important for retention.

In this paper, we focus on an influential mathematical model of pre-synaptic DA synthesis, release and reuptake [19]. The model can be used to investigate the effects of amounts of DA concentrations, enzyme expression levels, tyrosine inputs, dopamine transporters and firing rate changes (Fig. 1). Most of the model parameters are comparable to that in experiments (see [19] for details). However, the model consists of several coupled nonlinear differential equations and mathematical functions and variables that may potentially respond with very different timescales and amplitudes. This poses a significant problem in reducing the model to make it computationally more efficient, and for developing it across multiple scales, e.g. from molecular to neuronal-circuit levels.

In this work, we analyze the various components of the model in [19] by systematically perturbing its variables (substrates) [28]. This allows us to categorize the relative importance of the model variables in relation to each other and to tease apart the relative timescales of the variables thus providing the conditions for model reduction.

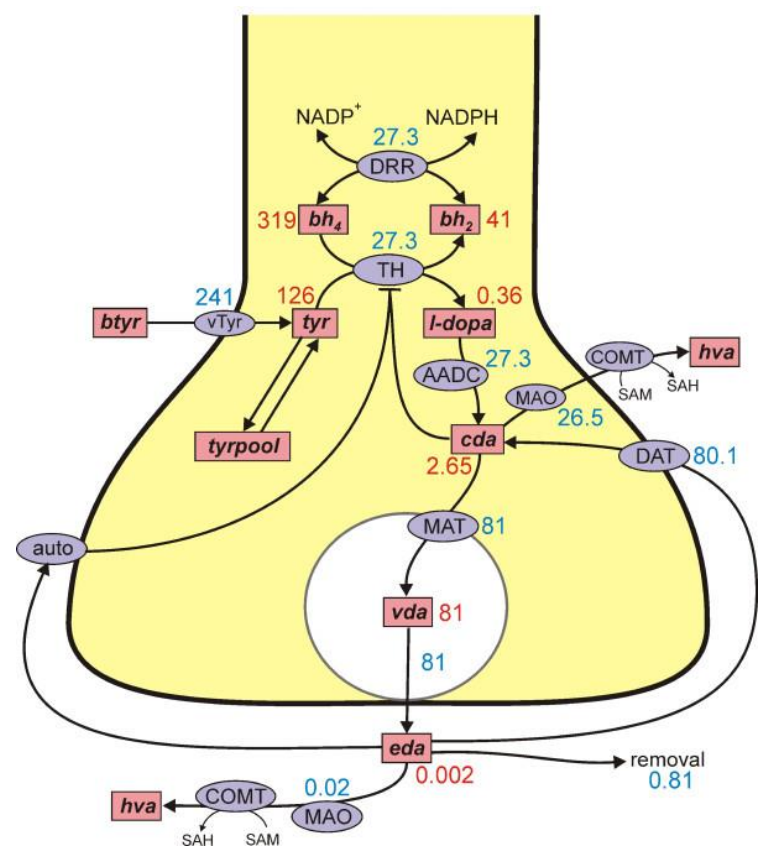

Fig. 1. Biochemical processes within a dopaminergic pre-synaptic terminal. Numbers denote steady-state concentrations of substrates (red) and fluxes/velocities (blue). Adapted from [19].

\section{COMPUTATIONAL MODEL OF DOPAMINE SYNTHESIS AND RELEASE}

The model in [19] consists of biochemical reactions that occur during the synthesis, release, catabolism and reuptake of DA within the presynaptic terminal. A schematic diagram of the model including these reactions is shown in Fig. 1. The model consists of nine coupled nonlinear differential equations that describe the chemical kinetics of the various substrates involved in maintaining homeostatic DA synthesis and release (see (1)-(9); Table I). The substrates are denoted in lower case while the enzyme names and velocities are denoted in upper case. Transport and reaction velocities are prefixed with a capital $V$ followed by the name of the enzyme, transporter or process in subscript. For example, the mathematical symbol $\mathrm{V}_{\mathrm{TH}}$ (tyr, bh4, cda, eda) denotes the velocity (function) of the tyrosine hydroxylase $(\mathrm{TH})$ reaction and its dependence on the concentrations of the substrates tyrosine (tyr), tetrahydrobiopterin (bh4), cytosolic DA (cda), and extracellular DA (eda). The full names for the rest of the substrates and velocities are shown in Table I.

Table I

Steady-state values for the substrates and velocities in the model.

\begin{tabular}{|c|c|c|c|}
\hline \multirow{2}{*}{$\begin{array}{l}\text { Substrate } \\
\text { /velocity }\end{array}$} & \multirow[b]{2}{*}{ Full name } & \multicolumn{2}{|c|}{ Steady state value } \\
\hline & & $\begin{array}{l}\text { Current full } \\
\text { model }\end{array}$ & [19] \\
\hline [bh2] & Dihydrobiopterin & 22.7 & 41 \\
\hline [bh4] & Tetrahydrobiopterin & 337.2 & 319 \\
\hline [1-dopa $]$ & 3,4-dihyroxyphenylalanine & 0.34 & 0.36 \\
\hline$[\mathrm{tyr}]$ & Tyrosine & 93.4 & 126 \\
\hline [tyr-pool] & Tyrosine pool & 701 & Not given \\
\hline [hva] & Homovanillic acid & 6.26 & Not given \\
\hline [cda] & Cystosolic dopamine & 4.2 & 2.65 \\
\hline [vda] & Vesicular dopamine & 78 & 81 \\
\hline [eda] & Extracellular dopamine & 0.012 & 0.002 \\
\hline $\mathrm{V}_{\mathrm{TH}}$ & Tyrosine hydroxylase & 26.7 & 27.3 \\
\hline $\mathrm{V}_{\mathrm{DRR}}$ & Dihydropteridine reductase & 26.7 & 27.3 \\
\hline $\mathrm{V}_{\text {tyrin }}$ & Neutral amino acid transporter & 241 & 241 \\
\hline$V_{\text {tyr-pool }}$ & Tyrosine pool & 26.7 & 27.3 \\
\hline $\mathrm{V}_{\mathrm{AADC}}$ & $\begin{array}{l}\text { Aromatic amino acid } \\
\text { decarboxylase }\end{array}$ & 77.5 & 81 \\
\hline $\mathrm{V}_{\mathrm{MAT}}$ & $\begin{array}{l}\text { Vesicular monoamine } \\
\text { transporter }\end{array}$ & 72.3 & 80.1 \\
\hline $\mathrm{V}_{\mathrm{DAT}}$ & Dopamine (reuptake) transporter & 0.12 & 0.02 \\
\hline $\mathrm{V}_{\text {catab }}$ & $\begin{array}{c}\text { Catabolism of cystosolic } \\
\text { dopamine }\end{array}$ & 22.7 & 41 \\
\hline
\end{tabular}

$$
\begin{aligned}
\frac{d[b h 2]}{d t}= & V_{\mathrm{TH}}(t y r, b h 4, c d a, e d a) \\
- & V_{\mathrm{DRR}}(b h 2, \mathrm{NADPH}, b h 4, \mathrm{NADP}) \\
\frac{d[b h 4]}{d t}= & V_{\mathrm{DRR}}(b h 2, \mathrm{NADPH}, b h 4, \mathrm{NADP}) \\
& -V_{\mathrm{TH}}(t y r, b h 4, c d a, e d a)
\end{aligned}
$$




$$
\begin{aligned}
\frac{d[t y r]}{d t}= & V_{\mathrm{TYRin}}(\operatorname{btyr}(t))-V_{\mathrm{TH}}(t y r, b h 4, c d a, e d a) \\
& -k_{1} \cdot[\mathrm{tyr}]+k_{-1} \cdot[\text { tyr }- \text { pool }]-\mathrm{k}_{\mathrm{tyr}}^{\mathrm{catab}} \cdot[\text { tyr }]
\end{aligned}
$$

$$
\begin{aligned}
\frac{d[l-d o p a]}{d t}= & V_{\mathrm{TH}}(t y r, b h 4, c d a, e d a) \\
& -V_{A A D C}(l-d o p a)
\end{aligned}
$$

$$
\begin{aligned}
\frac{d[c d a]}{d t}= & V_{A A D C}(l-d o p a)-V_{M A T}(c d a, v d a) \\
& +V_{D A T}(e d a)-k_{c d a}^{c a t a b} \cdot c d a
\end{aligned}
$$

$\frac{d(v d a)}{d t}=V_{M A T}(c d a, v d a)-$ fire $(t) \cdot v d a$

$$
\begin{aligned}
\frac{d(e d a)}{d t}= & f i r e(t) \cdot v d a-V_{D A T}(e d a) \\
& -V_{C A T A B}(e d a)-k_{r e m} \cdot e d a
\end{aligned}
$$

$$
\begin{aligned}
\frac{d(h v a)}{d t} & =k_{c d a}^{c a t a b} \cdot c d a+V_{C A T A B}(e d a) \\
& -k_{h v a}^{c a t a b} \cdot h v a
\end{aligned}
$$

$$
\begin{aligned}
\frac{d(\text { tyrpool })}{d t} & =k_{1} \cdot \text { tyr }-k_{-1} \cdot \text { tyrpool } \\
& -k_{\text {tyrpool }}^{\text {catab }} \cdot \text { tyrpool }
\end{aligned}
$$

The function fire(t) in (6) and (7) is related to the firing rate of the pre-synaptic DA neurons and is generally time dependent [19]. In this paper, we follow [19] and set the value to be $1 \mu \mathrm{M} / \mathrm{h} r$, i.e. vesicular dopamine is released at a constant rate with entire pool turning over every hour. The specific functional forms of the reaction velocities used for (1)-(9) are determined by Michaelis-Menten kinetics as follows [19]:

$$
\begin{aligned}
& V_{T H}(t y r, b h 4, e d a, c d a)= \\
& \left(\frac{0.56}{1+\left(\frac{[t y r]}{K_{i(t y r)}}\right)}\right) \cdot\left(\frac{4.5}{\left(8\left(\frac{[e d a]}{0.002024}\right)^{4}+1\right)}+0.5\right) \\
& \cdot\left(\frac{V_{\max } \cdot[t y r] \cdot[b h 4]}{[t y r][b h 4]+K_{t y r}[b h 4]+K_{t y r} K_{b h 4}\left(1+\left(\frac{[c d a]}{K_{i(c d a)}}\right)\right)}\right) \\
& V_{\text {tyrin }}(b t y r)=\frac{400[\text { btyr }]}{64+[\text { btyr }]}
\end{aligned}
$$

$V_{D R R}(b h 2, N A D P H, b h 4, N A D P)=$
$\left(\frac{V_{\max }^{f} \cdot[b h 2] \cdot N A D P H}{\left(k_{m}^{b h 2}+[b h 2]\right)\left(k_{m}^{N A D P H}+N A D P H\right)}\right)-\left(\frac{V_{\max }^{b} \cdot[b h 4] \cdot N A D P}{\left(k_{m}^{b h 4}+[b h 4]\right)\left(k_{m}^{N A D P}+N A D P\right)}\right)$

$$
\begin{aligned}
& V_{A A D C}(l-\text { dopa })=\frac{V_{A A D C, \max } \cdot[l-d o p a]}{k_{A A D C, m}+[l-d o p a]} \\
& V_{M A T}(c d a, v d a)=\frac{V_{M A T, \max } \cdot[c d a]}{k_{M A T, m}+[c d a]}-k_{\text {out }} \cdot[v d a] \\
& V_{D A T}(e d a)=\frac{V_{D A T, \max } \cdot[e d a]}{k_{D A T, m}+[\text { eda }]} \\
& V_{c a t a b}(e d a)=\frac{V_{c a t a b, \max } \cdot[e d a]}{k_{c a t a b, m}+[e d a]}
\end{aligned}
$$

The kinetic parameters (with units in $\mu \mathrm{M}, \mu \mathrm{M} / \mathrm{hr}$ ) are taken from [19] with the exception of those denoted by an * which are from [27]: $\mathrm{k}_{\mathrm{AADC}, \mathrm{m}}=130, \mathrm{~V}_{\mathrm{AADC}, \max }=10000, \mathrm{k}_{\mathrm{DAT}, \mathrm{m}}=0.2$, $\mathrm{V}_{\mathrm{DAT} \text { max }}=8000, k_{m}^{b h 2}=100, V_{D R R, \text { max }}^{f}=200, k_{m}^{b h 4}=10$, $k_{m}^{N A D P}=k_{m}^{N A D P H}=75, V_{\text {max }}^{b}=80, k_{M A T, m}=3, V_{M A T, \max }=$ $7082, k_{\text {out }}=40, K_{\text {tyr }}=46, K_{\text {bh } 4}=60, V_{\text {max }}=125, K_{i(c d a)}=$ $110, K_{i(t y r)}=150, \mathrm{NADPH}=124^{*}, \mathrm{NADP}=0.25^{*}, k_{1}=6$, $k_{-1}=0.6, k_{\text {tyr-pool }}^{\text {catab }}=0.2, k_{\text {tyr }}^{\text {catab }}=0.2, k_{\text {cda }}^{\text {catab }}=10$, $k_{\text {catab,m }}=3, V_{\text {catab,max }}=30, k_{\text {hva }}^{\text {catab }}=3.45$, and $k_{\text {rem }}=400$.

The initial values for nicotinamide adenine dinucleotide phosphate (NADPH) and 2-oxoaldehyde dehydrogenase $(\mathrm{NADP}+)$ are not readily provided in [19] and are obtained from [28], taken as 124 um and 0.25 , respectively. The ratio of NADPH to NADP+ is described as 500:1 [29], consistent with those used in this model. Although the initial parameter values used in this model vary slightly from those outlined in [19], but still within the ranges of experimental values (see [19]), the final steady-state values are readily consistent with those in the original model (Table I).

The full model is simulated in MATLAB (MATLAB R2013b, The MathWorks, Natick, MA) with Euler's numerical scheme being used for integrating the differential equations. The time step size used for numerical integration in the model is $0.0036 \mathrm{~s}(0.00001 \mathrm{~h})$.

\section{RESULTS}

Intrinsically fast and slow dependent variables or substrates were elucidated by carrying out step perturbation of each variable and substrate while analyzing the state of the full model at each step. This 'separation of timescales' approach can allow the model to be reduced for increased computational speed allowing the user to investigate either slow or fast dynamics independently with greater efficacy [28]. For example, if fast timescale (e.g. order of milliseconds) is the focus of study, the much slower variables can be assumed to be relatively constant, and are converted from differential equations to mere mathematical functions or constants. The model is then reduced to a 'fast mode' requiring reduced computational cost. Similarly, if slow timescale (e.g. order of hours) is the focus, the much faster variables are assumed to have rapidly reached their quasi-steady states, i.e. they become 
mathematical functions. As a result we can have two approximate models; one that can be used to examine fast dynamics and the other for slower dynamics. Further reduction can be obtained by observing how one substrate's relative influence on another. For example, if perturbing one substrate elicits a small change in the amplitude (concentration level) of another substrate, then we could ignore the contribution of the relationship between these two substrates, e.g. replace by a constant parameter. To search for the relative timescales and effects of one substrate affected by another, we resort to perturbation of the variables as discussed below.

\section{A. Model Perturbation and Categorization}

A simple yet robust method of perturbation is used to categorize variable responses and timescales. The whole system is first simulated and allowed to reach a steady state. Perturbations of each substrate/variable (e.g. tyr) are then applied by abruptly changing one of the depended substrates/variables (e.g bh4, cda or eda) in a step-wise manner (Fig. 2) [28]. The system is then allowed to reach its new steady state during the perturbed phase. Assuming a substrate increases exponentially towards a new steady state following perturbation, we find the amount of time $(\tau)$ it takes for the dependent variable to reach $67 \%$ (33\% in cases of exponential decay) of the new steady state (Fig. 2). If $\tau$ for that particular variable is relatively fast, the relative variable or substrate is categorized as a fast variable. Similarly if $\tau$ is slow, the variable/substrate is categorized as slow. Variables and substrates that display intermediate timescales or with both fast and slow dynamics are categorized as "intermediate/mixed". This process is repeated for other substrates/variables with different perturbing amplitudes (1.5, 2 and 3 times the respective steady-state value), and the overall results are shown in Tables II-V.

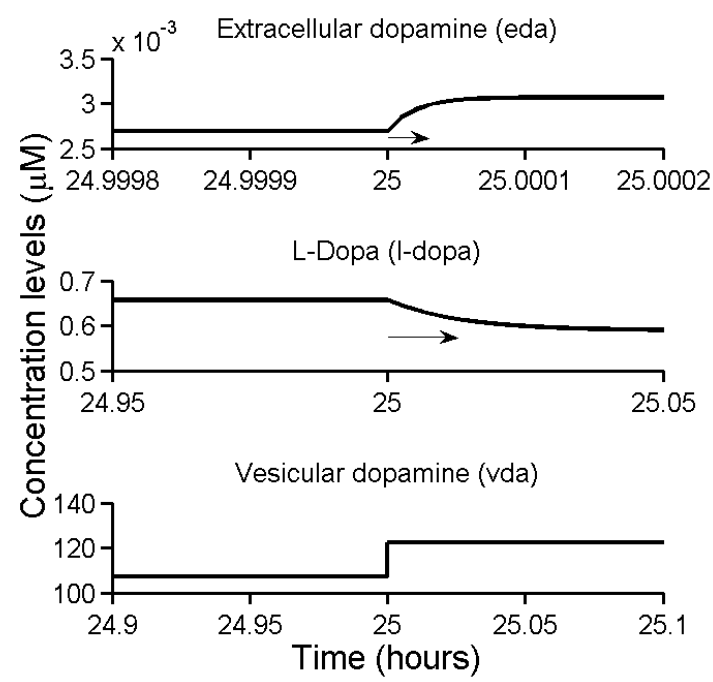

Fig. 2. Example of responses of the variables/substrates (extracellular dopamine, eda, and L-Dopa, l-dopa) over time during a step perturbation of another variable/substrate (vesicular dopamine, vda). Arrows denote the evaluated timescales of eda and 1-dopa. Note the different the scales on the horizontal and vertical axes. Perturbation occurs from the $25^{\text {th }}$ hour onward.
Table II shows the relative changes in steady-state values for the perturbation of all variables and substrates, while Table III reveals coupling strengths based on the perturbations. Clearly, the relative change and timescale can vary greatly from one variable to another. For example, in terms of percentage change [1-dopa] seems to depend more on [tyr] than [vda] and [cda], while [tyr-pool] is more sensitive to [tyr] compared to [1-dopa] to [tyr]. In terms of timescales, [cda] responds to perturbation of [vda] with about two orders of magnitude than [1-dopa] responding to the same [vda] perturbation. Table IV shows all the variables/substrates dynamics as having intrinsically slow, fast or intermediate/mixed dynamics, and they are categorized in Table V.

Table II

PERCENTAGE CHANGE IN STEADY STATES OF SUBSTRATES/VARIABLES AFTER PERTURBATION

\begin{tabular}{|c|c|c|c|c|}
\hline \multirow{2}{*}{ 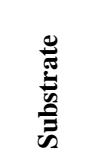 } & \multirow{2}{*}{ 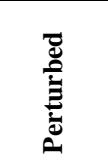 } & \multicolumn{3}{|c|}{ Perturbation Amplitude (\%) } \\
\hline & & 1.5 & 2 & 3 \\
\hline bh2 & tyr & 4.0 & 6.4 & 14.47 \\
\hline bh2 & bh4 & 0.64 & 1.007 & 1.3732 \\
\hline bh2 & cda & -28.7458 & -37.5458 & -43.7729 \\
\hline bh2 & eda & -41.5751 & -49.3284 & -52.3345 \\
\hline bh4 & bh2 & 30.624 & 44.8121 & 74.8 \\
\hline bh4 & tyr & 4.61 & 7.07 & 10.05 \\
\hline bh4 & cda & -0.86 & -2.79 & -3.4 \\
\hline bh4 & eda & 2.45 & 5.34 & 6.85 \\
\hline 1-dopa & tyr & 12.02 & 17.09 & 22.197 \\
\hline 1-dopa & bh4 & 1.82 & 2.63 & 3.38 \\
\hline 1-dopa & cda & 2.70 & 7.892 & 11.06 \\
\hline 1-dopa & vda & -9.4658 & -9.42 & -32.9072 \\
\hline cda & 1-dopa & 72.98 & 17.889 & 39.339 \\
\hline cda & $\mathrm{vda}$ & -30.757 & -83.136 & -99.8 \\
\hline cda & eda & 11.14 & 23 & 58.48 \\
\hline vda & cda & 7.67 & 3.0755 & 17.19 \\
\hline eda & vda & 12.292 & 34.0633 & 56.19 \\
\hline hva & cda & 3.02 & 8.02 & 14.81 \\
\hline tyr-pool & tyr & 55.24 & 67.3941 & 70.1628 \\
\hline tyr & tyr-pool & 70.172 & 80.93 & 70.172 \\
\hline tyr & bh4 & -43.68 & -80.94 & -235.38 \\
\hline tyr & cda & 63.15 & 62.5437 & 62.0258 \\
\hline tyr & eda & 37.78 & 38.425 & 48.6651 \\
\hline
\end{tabular}

Table III

CLASSIFICATION OF SUBSTRATES/VARIABLES' COUPLING STRENGTHS

\begin{tabular}{|l|l|l|lll|}
\hline \multicolumn{7}{|c|}{ Strong } \\
\hline tyrpool $\rightarrow$ tyr & 1-dopa $\rightarrow$ cda & eda $\rightarrow$ bh2 \\
\hline vda $\rightarrow$ cda & bh2 $\rightarrow$ bh4 & cda $\rightarrow$ bh2 \\
\hline \multicolumn{7}{|c|}{ Weak } \\
\hline bh4 $\rightarrow$ bh2 & cda $\rightarrow$ bh4 & bh4 $\rightarrow$ 1-dopa \\
\hline \multicolumn{7}{|c|}{ Intermediate } \\
\hline tyr $\rightarrow$ bh2 & eda $\rightarrow$ bh4 & eda $\rightarrow$ tyr \\
\hline tyr $\rightarrow$ 1-dopa & cda $\rightarrow$ vda & eda $\rightarrow$ bh4 \\
\hline
\end{tabular}




\begin{tabular}{|ll|lll|lll|}
\hline cda & $\rightarrow$ 1-dopa & eda & $\rightarrow$ & vda & eda & $\rightarrow$ & cda \\
\hline vda $\rightarrow$ 1-dopa & cda & $\rightarrow$ & tyr & cda & $\rightarrow$ & hva \\
\hline vda $\rightarrow$ eda & tyr & $\rightarrow$ & tyrpool & bh4 & $\rightarrow$ & tyr \\
\hline
\end{tabular}

Table IV

RISE AND DECAY TIME CONSTANTS ( $\tau$ ) (IN UNITS OF $10^{-5}$ HOURS) OF SUBSTRATES/VARIABLES AFTER PERTURBATIONS

\begin{tabular}{|c|c|c|c|c|}
\hline \multirow{2}{*}{ 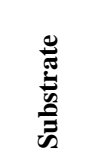 } & \multirow{2}{*}{ 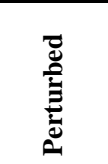 } & \multicolumn{3}{|c|}{ Perturbation Amplitude } \\
\hline & & 1.5 & 2 & 3 \\
\hline bh2 & tyr & 0.097 & 0.096396 & 0.094077 \\
\hline bh2 & bh4 & 0.332531 & 0.324372 & 0.325767 \\
\hline bh2 & cda & 0.182653 & 0.178864 & 0.176167 \\
\hline bh2 & eda & 0.17579 & 0.17278 & 0.17171 \\
\hline bh4 & bh2 & 1.781210 & 8.197055 & 12.66077 \\
\hline bh4 & tyr & 0.058466 & 0.063716 & 0.64047 \\
\hline bh4 & cda & 0.129081 & 0.127165 & 0.126272 \\
\hline bh4 & eda & 0.125855 & 0.12221 & 0.12308 \\
\hline 1-dopa & tyr & 0.099120 & 0.099502 & 0.099872 \\
\hline 1-dopa & bh4 & 0.088901 & 0.090077 & 0.092284 \\
\hline 1-dopa & cda & 0.064116 & 0.078224 & 0.081265 \\
\hline 1-dopa & vda & 0.038861 & 0.050756 & 0.114403 \\
\hline cda & 1-dopa & 0.143980 & 0.125000 & 0.102000 \\
\hline cda & vda & 0.000323 & 0.001769 & 0.009089 \\
\hline cda & eda & 0.064515 & 0.16417 & 0.057020 \\
\hline vda & cda & 0.002426 & 0.002426 & 0.002429 \\
\hline eda & vda & 0.059719 & 0.051678 & 0.037623 \\
\hline hva & cda & 0.028875 & 0.028845 & 0.028832 \\
\hline tyr-pool & tyr & 0.124305 & 0.124000 & 0.12500 \\
\hline tyr & tyr-pool & 0.012233 & 0.01190 & 0.021202 \\
\hline tyr & bh4 & 0.62187 & 0.60798 & 0.60286 \\
\hline tyr & cda & 0.757081 & 0.753788 & 0.757526 \\
\hline tyr & eda & 0.75229 & 0.75692 & 0.768391 \\
\hline
\end{tabular}

Table V

CLASSIFICATION OF SUBSTRATES/VARIABLES' TIMESCALES

\begin{tabular}{|c|c|c|}
\hline Fast & Slow & Intermediate/Mixed \\
\hline eda & bh4 & bh2 \\
\hline vda & tyr & 1-dopa \\
\hline hva & tyr-pool & cda \\
\hline
\end{tabular}

\section{B. Towards Reducing Models with Fast or Slow Modes}

To obtain a reduced slow model, fast substrates will have to be assumed to have rapidly reached their steady states and their associated differential equations will be transformed into functions i.e. at their steady state values. For example, we can set the differential equation (7) for the fast substrate eda, $\mathrm{d}[\mathrm{eda}] / \mathrm{dt}=0$, solve the resultant algebraic equation (using the Mathematica software package) [28], and obtain the following:

$$
\begin{aligned}
& {[e d a]=} \\
& \frac{\text { fire }(t) \cdot \text { release } \cdot k_{m}^{\text {catab }, e d a} \cdot v d a \cdot k_{D A T, m}}{\left(k_{\text {rem }} \cdot k_{m}^{\text {catab,eda }}+V_{\max }^{\text {catab,eda }}\right) \cdot k_{D A T, m}+k_{m}^{\text {catab,eda }} \cdot V_{D A T, \max }}
\end{aligned}
$$

Similarly for the other fast variables vda and hva. If we instead wish to obtain a reduced fast model, then the slow substrates can be assumed to be approximately constant (with specific steady-state values initially obtained from the full model simulations), and similar solving of algebraic equations will be required. Specifically, we could keep the dynamics of the fast variables eda, vda and hva, and set the rest of slow and intermediate/mixed variables to be constant [28]. For example, if we have set $\mathrm{d}[1-\mathrm{dopa}] / \mathrm{dt}=0$ in (4), we would have

$[l-d o p a]=\frac{V_{T H}(t y r, b h 4, c d a, e d a) \cdot k_{A A D C, m}}{V_{A A D C, \max }-V_{T H}(t y r, b h 4, c d a, e d a)}$

From our simulations, we find that the steady states of the full and reduced model are very similar (not shown).

Further reduction of the model can be achieved by observing the relative effect of one substrate over another. For example, [cda] has relatively smaller effect on [bh4] (Table III), and we can ignore this factor in the model reduction process. Based on this, as [bh4] depends on [cda] only through $\mathrm{V}_{\mathrm{TH}}$ (see (2)), then $\mathrm{V}_{\mathrm{TH}}$ can be reduced from (10) to a simpler function:

$$
\begin{aligned}
& V_{T H}(t y r, b h 4, e d a)= \\
& \left(\frac{0.56}{1+\left(\frac{[t y r]}{K_{i(t y r)}}\right)}\right) \cdot\left(\frac{4.5}{\left(8\left(\frac{[e d a]}{0.002024}\right)^{4}+1\right)}+0.5\right) \\
& \left(\frac{V_{\max } \cdot[t y r] \cdot[b h 4]}{[t y r][b h 4]+K_{t y r}[b h 4]+K_{t y r} K_{b h 4} \alpha}\right)
\end{aligned}
$$

where $\alpha$ is some constant parameter. If we have made a further approximation that the influence of [eda] on [bh4] is relatively weak, we can further simplify it to:

$V_{T H}(t y r, b h 4)=$

$\beta \cdot\left(\frac{0.56}{1+\left(\frac{[t y r]}{K_{i(t y r)}}\right)}\right) \cdot\left(\frac{V_{\max } \cdot[t y r] \cdot[b h 4]}{[t y r][b h 4]+K_{t y r}[b h 4]+K_{t y r} K_{b h 4} \alpha}\right)$

for some constant parameter $\beta$.

To obtain a reduced slow model, we set the fast variables eda, vda and hva to be instantaneous while keeping the dynamics of the slow and intermediate/mixed variables. The steady states of the reduced model are similar to that in the full model (not shown). The execution time for a 48 hour simulation of the reduced slow model and the full model are compared using MATLAB's stopwatch timer. By comparing the computational speed of a single run or realization of simulated 48 hours, we found that the reduced slow model 
with fewer differential equations is 0.706 seconds faster than the full model. If we repeat the simulations over 10,000 runs (e.g. in search for optimal drug dosage), then we can save about 1.96 hours of computational time.

To obtain a reduced fast model, reactions that occur on the scale of milliseconds to tens of seconds are isolated and analyzed by either holding substrates with slower dynamics at a constant value or calculating their values as functions at each time step. Homovanillic acid (hva) serves as an endpoint for the catabolism of cytosolic dopamine (cda) and exhibits fast dynamics. As this model does not explicitly simulate the catabolism of cda, no other substrates are dependent on hva, therefore it can be excluded from the analysis. The fast model can thus be reduced further by simulating the dynamics of only eda and vda (extracellular and vesicular dopamine) with just two differential equations and the control parameter for neuronal spiking, 'fire'.

The constant values for the 'slow' substrates can be obtained by simulating either the full or reduced slow model until the difference between the value of substrate $X$ at time $T$, $(\mathrm{X}(\mathrm{T}))$, and $\mathrm{X}(\mathrm{T}-1)$ is less than $\varepsilon$, where $\varepsilon$ is small enough to represent no significant change. In our simulations, we select $\varepsilon$ to be 0.002 . We found that the steady states of the reduced model are similar to that in the full model (not shown). Furthermore, with fewer differential equations, the reduced fast model was able to complete a 48 hour simulation 2.6064 seconds faster than the original full model. If the simulation were to be repeated 10,000 times we would save 7.24 hours using the reduced spiking model.

\section{CONCLUSION}

We have analyzed an influential computational model of DA synthesis and release using a simple perturbation method. This method has previously been applied successfully to a serotonergic pre-synaptic terminal [28]. In this work, we show similar success for the dopaminergic pre-synaptic terminal but with a different set of substrates and reactions. Specifically, we show that different variables or substrates are affected (or perturbed) much more than others. Moreover, the intrinsic timescales of the variables or substrates can vary widely, ranging across several orders of magnitude. The classification of timescales is also different from our previous work. This large range of timescales can potentially permit us to separate the slow and fast variables and hence approximate the model into a slow or fast version. Indeed, in this work, we have successfully teased apart the multiscale processes in the model. In particular, although we have adopted a rather simple perturbation method, the reduced models have similar steady states as the full model, while their computational efficiencies are significantly enhanced due to fewer differential equations. Further work would include exploring more advanced perturbation techniques, e.g. simultaneous perturbations of variables/substrates, and other nonlinear model reduction methods.

It should be noted that the reduced models in this work are still equipped for testing the effects of in-silico drug manipulation on targets such as the dopamine transporter
(DAT), MAO-A and MAO-B related metabolism, L-Dopa uptake, and autoreceptor function, thus providing a useful and efficient platform for faster drug discovery and development. Reduced models of neuromodulators can be incorporated into large-scale computational models [21]-[24], [30]-[32]. In particular, our previous 'fast' serotonergic pre-synaptic terminal model has been shown to integrate successfully into a spiking neuronal network model, effectively simulating an entire population of serotonergic neurons [28]. We would expect similar success for the reduced dopaminergic model presented in this work to bridge from molecular to cognitive levels [21]-[22].

\section{REFERENCES}

[1] K.A. Neve., J.K. Seamans, and H. Trantham-Davidson, "Dopamine receptor signaling”. J. Recet. Signal Transduct. Res., vol. 24, pp. 165-205, 2004.

[2] C. Missale, S.R. Nash, S.W. Robinson, M. Jaber, and M.G. Caron, "Dopamine receptors: from structure to function." Physiol. Rev., vol. 78, pp. 189-225, 1998.

[3] P.W. Glimcher, "Understanding dopamine and reinforcement learning: the dopamine reward prediction error hypothesis," Proc. Nat. Acad. Sci., vol. 108, pp. 15647-15654, 2011.

[4] E.E. Steinberg, R. Keiflin, J.R. Boivin, I.B. Witten, K. Deisseroth, and P.H. Janak, "A causal link between prediction errors, dopamine neurons and learning," Nat. Neurosci., vol. 16, pp. 966-973, 2013.

[5] R.J. Baldessarini, F.I. Tarazi, "Drugs and the treatment of psychiatric disorders". in The Pharmacologic Basis of Therapeutics , J.G. Hardman, L.E. Limbird, Eds. McGraw-Hill, New York, 2001, pp. 485-520.

[6] C.G. Goetz "Dopaminergic agonists in the treatment of Parkinson's disease". Neurol., vol. 40, pp. 50-54, 1990.

[7] P. Damier, E.C. Hirsch, Y. Agid, and A.M. Graybriel, "The substantia nigra of the human brain II. Patterns of loss of dopamine-containing neurons in Parkinson's disease," Brain, vol. 122, 1437-1448, 1999.

[8] M. Laruelle, A. Abi-Dargham, R. Gil, L. Kegeles, and R. Innis, "Increased dopamine transmission in schizophrenia: relationship to illness phases," Biol. Psychiatry, vol. 46, pp. 11437-144856-144872, 1999.

[9] H. Tost, T. Alam, and A. Meyer-Lindenberg. "Dopamine and psychosis: theory, pathomechanisms and intermediate phenotypes," Neurosci. Biobehav. Rev., vol. 34, pp. 689-700, 2010.

[10] M. El Mansari, B.P. Guiard, O. Chernoloz, R. Ghanbari, N. Katz, and P. Blier, "Relevance of norepinephrine-dopamine interactions in the treatment of major depressive disorder," CNS Neurosci. Ther., 16(3): e1-17, 2010. doi: 10.1111/j.1755-5949.2010.00146.x.

[11] A. Bonci, G. Bernardi, P. Grillner, and N.B. Mercuri, "The dopaminecontaining neuron: maestro or simple musician in the orchestra of addiction," Trends Pharmacol. Sci., vol. 24, pp. 172-177, 2003.

[12] N.D. Volkow, J.S. Fowler, G.J. Wang, R. Baler, and F. Telang, "Imaging dopamine's role in drug abuse and addiction," Neuropharmacology, vol. 56, Suppl 1, pp. 3-8, 2009.

[13] D.J. Brooks, "Dopamine agonists: their role in the treatment of Parkinson's disease," J. Neurol. Neurosurg. Psychiatry, vol. 68, pp. 685-689, 2000.

[14] R. Pivonello, D. Ferone, W.W. de Herder, J.M. Kros, M.L. De Caro, M. Arvigo, L. Annunziato, G. Lombardi, A. Coalo, L.J. Hofland, and S.W. Lamberts, "Dopamine receptor expression and function in corticotroph pituitary tumors," J. Clin. Endocrin. Metab., vol. 89, pp. 2452-2462, 2004.

[15] R. Scranton, and A. Cincotta, "Bromocriptine-unique formulation of a dopamine agonist for the treatment of type 2 diabetes," Expert Opin. Pharmacother., vol. 11, pp. 269-279, 2010.

[16] C.B. Aiken, "Pramipexole in psychiatry: a systematic review of the literature," J. Clin. Psychiatry, vol. 68, pp. 1230-1236, 2007. 
[17] J.-M. Beaulieu, and R.R. Gainetdinov, "The physiology, signaling, and pharmacology of dopamine receptors." Pharmacol. Rev., vol. 63, pp. 182-217, 2011.

[18] Z. Qi, G.W. Miller, and E.O. Voit, "Computational systems analysis of dopamine metabolism," PLoS One, 3(6): e2444, 2008. doi: 10.1371/journal.pone.0002444.

[19] J.A. Best, H.F. Nijhout, and M.C. Reed, "Homeostatic mechanisms in dopamine synthesis and release: a mathematical model," Theor. Biol. Med. Model., 6:21, 2009. doi: 10.1186/1742-4682-6-21.

[20] M. Reed, H.F. Nijhout, J. Best, Mathematical insights into the effects of levodopa, Front. Integr. Neurosci., 6:21, 2012. doi: 10.3389/fnint.2012.00021.

[21] P.Eckhoff, K.F. Wong-Lin, and P. Holmes, "Optimality and robustness of a biophysical decision-making model under norepinephrine modulation," $\mathrm{J}$. Neurosci., vol. 29, pp. 4301-4311, 2009.

[22] P. Eckhoff, K. Wong-Lin, and P. Holmes, "Dimension reduction and dynamics of a spiking neural network model for decision making under neuromodulation," SIAM J. Appl. Dyn. Syst., vol. 10, pp. 148-188, 2011.

[23] D.H. Wang, and K. Wong-Lin, "Comodulation of dopamine and serotonin on prefrontal cortical rhythms: a theoretical study," Front. Integr. Neurosci, 7:54, 2013. doi: 10.3389/fnint.2013.00054.

[24] M.C. Reed, H.F. Nijhout, J. Best, "Computational studies of the role of serotonin in the basal ganglia," Front. Integr. Neurosci., 7:24, 2013. doi: 10.3389/fnint.2013.00041.

[25] B. Lo and M.J. Field, " Conflict of Interest in Medical Research Education and Practice," National Academy Press, 2009.

[26] S.S. Ou-Yang , J.Y. Lu , X.Q. Kong, Z.J. Liang , C. Luo, and H. Jiang, "Computational drug discovery," Acta. Pharmacol. Sin., vol. 33, pp.1131 1140, 2012.

[27] P.V. Kokotovic, J.J. Allenmong, J.R. Winelman, and J.H. Chow, "Singular perturbation and iterative separation of time scales," Automatica, vol. 16, pp. 23-33, 1980.

[28] G. Flower, and K. Wong-Lin, "Reduced computational models of serotonin synthesis, release and reuptake, IEEE Trans. Biomed. Eng., vol. 61, pp. 1054-1061, 2014.

[29] R.L Veech, L.V. Eggleston, and H.A. Krebs, "The redox state of free nicotinamide-adenine dinucleotide phosphate in the cytoplasm of rat liver," Biochem. J., vol. 115, pp. 609-619, 1969.

[30] J. Jalewa, A. Joshi, T.M. McGinnity, G. Prasad, K. Wong-Lin, and C. Hölscher, "Neural circuit interactions between the dorsal raphe nucleus and the lateral hypothalamus: an experimental and computational study," PLoS One, 9(2): e88003, 2014. doi: 10.1371/journal.pone.0088003.

[31] K. Wong-Lin, A. Joshi, G. Prasad, and T.M. McGinnity, "Network properties of a computational model of the dorsal raphe nuclues," Neural Netw., vol. 32, pp. 15-25, 2012.

[32] A. Joshi, K. Wong-Lin, T.M. McGinnity, and G. Prasad, "A mathematical model to explore the interdependence between the serotonin and orexin/hypocretin systems," in Proc. IEEE Eng. Med. Biol. Soc. Conf., 2011, pp. $7270-7273$. 